OAK RIDGE NATIONAL LABORATORY

operated by

UNION CARBIDE CORPORATION

NUCLEAR DIVISION

for the

U.S. ATOMIC ENERGY COMMISSION

$$
\begin{aligned}
& \text { ORNL- TM- } 1890 \\
& \text { CONF- } 670905 \ldots-8
\end{aligned}
$$

W.C. Robinson, F.H. Patterson

J.D. Fleming and C.W. Gorton 


\section{DISCLAIMER}

This report was prepared as an account of work sponsored by an agency of the United States Government. Neither the United States Government nor any agency Thereof, nor any of their employees, makes any warranty, express or implied, or assumes any legal liability or responsibility for the accuracy, completeness, or usefulness of any information, apparatus, product, or process disclosed, or represents that its use would not infringe privately owned rights. Reference herein to any specific commercial product, process, or service by trade name, trademark, manufacturer, or otherwise does not necessarily constitute or imply its endorsement, recommendation, or favoring by the United States Government or any agency thereof. The views and opinions of authors expressed herein do not necessarily state or reflect those of the United States Government or any agency thereof. 


\section{DISCLAIMER}

Portions of this document may be illegible in electronic image products. Images are produced from the best available original document. 


\section{LEGAL NOTICE -}

This report was prepared as an account of Government sponsored work. Neither the United States, nor the Cammission, nor any person acting on behalf of the Commissiun:

A. Makes any warranty or representation, expressed or implied, with respect to the accuracy, completeness, or usefulness of the information contained in this report, or that the use of any information, apparatus, method, or process disclosed in this report may not infringe privafely owned rights; or

B. Assumes any liabilities with respect to the use of, or for damages resulting from the use of any information, apparatus, method, or process disclosed in this report.

As used in the above, "person acting on behalf of the Commission" includes any employee or contractor of the Commission, or employee of such contractor, to the extent that such employee or contractor of the Commission, or employee of such contractur prepares, disseminates, or provides access 10, any information pursuant to his employment or contract with the Commission, or his employment with such controctor. 


\title{
COII PRICAS
}

Contract No. W-7405-eng-26

H. 3.00

$\mathrm{MT}$

\section{METAIS AND CERAMICS DIVISION}

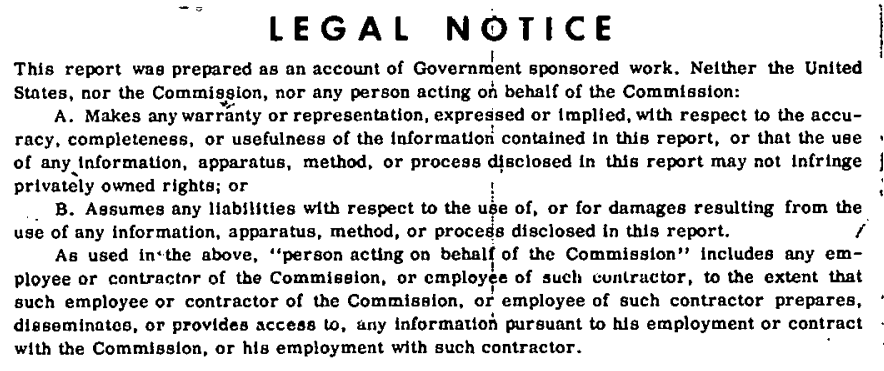

CHEMICAL VAPOR DEPOSITION STATISTICAL PARAMEIRIC STUDY

W. C. Robinson, F. H. Patterson,

J. D. Fleming, and C. W. Gorton

Paper for Conference on Chemical Vapor Deposition of Refractory Metals, Alloys and Compounds, Gatlinburg, Tennessee, September 12-13, 1967

\section{JULY 1967}

\author{
OAK RIDGE NATIONAL LABORATORY \\ Oak Ridge, Tennessee \\ operated by \\ UNION CARBIDE CORPORATION \\ for the \\ U.S. ATOMIC ENERGY COMMISSION
}




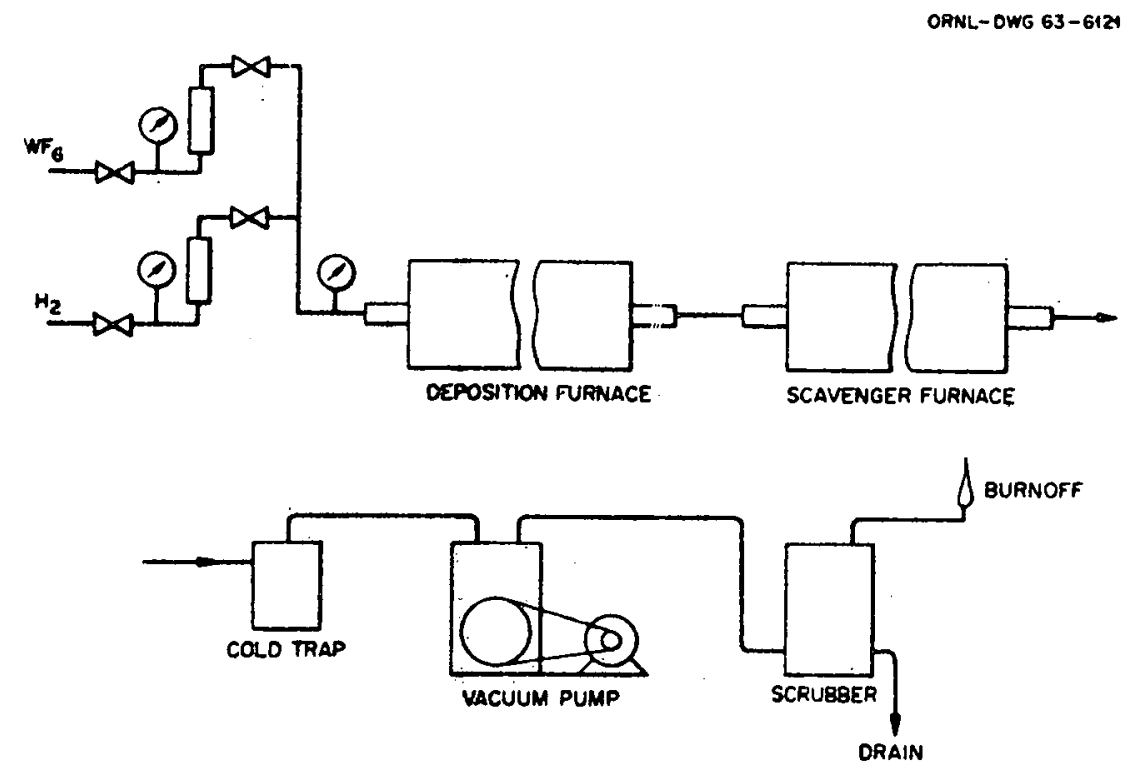


Abstract ... . . . . . . . . . . . . . . . .

Introduction . . . . . . . ................ I 1

Experimeńtal Apparatus . . . . . . . . . . . . . . 2

Statistical Program ..................... . . 3

Procedure and Experimental Results . . . . . . . . . . . 4

Statistical Results . . . . . . . . . . ..... 12

Discussion and Interpretation . . . .............. 17

Conclusions and Future Work . . . . . . . . . . . . . 21

Acknowledgments . . . . . . . . . . . . . . . 21 


\title{
W. C. Robinson, F. H. Patterson ${ }^{1}$ \\ J. D. Fleming, ${ }^{2}$ and C. W. Gorton ${ }^{2}$
}

\begin{abstract}
Factors affecting the chemical vapor deposition of tungsten are being evaluated in a statistically designed parametric study. Eighty-one experiments were required in the $\mathrm{WF}_{6}-\mathrm{H}_{2}$ deposition system to provide a third-order polynomial correlation between the response parameters and the independent system parameters over the experimental range of 450 to $820^{\circ} \mathrm{C}$, 9 to 20 torrs, 60 to $370 \mathrm{~cm}^{3} \mathrm{WF}_{6}$ per minute, and 752 to $5240 \mathrm{~cm}^{3} \mathrm{H}_{2}$ per minute. The system parameters were $\mathrm{H}_{2} / \mathrm{WF}_{6}$ ratio, total flow rate, temperature, and pressure. The initial response parameters were determined for an internal deposit in a 15-in. length of a 0.807-in.-ID copper mandrel. The application and limitations of utilizing the resulting curves for systems of different size and geometry will be discussed. The deposition rate of tungsten at hydrogen fluoride mole fractions of $0.02,0.05,0.10$, and 0.20 was also determined for each experiment and correlated with the system parameters. These data in conjunction with theoretical consideration of the system give indications of the rate-controlling process in portions of the experimental range. The agreement between theory and experiment and a preliminary interpretation of the deposition mechanism at various conditions will be presented.
\end{abstract}

\section{INTRODUCTION}

Chemical vapor deposition is being investigated at Oak Ridge National Laboratory as a possible fabrication method for hardware that is otherwise difficult to fabricate. These items, including fuel, tubing, coatings, and special parts, are often of an intricate shape. Furthermore, very close control of total thickness, efficiency, and thickness uniformity are often necessary for successful fabrication of the desired component: The

\footnotetext{
${ }^{1}$ Now at Battelie Memorial Institute, Columbus, Ohio.

${ }^{2}$ Georgia Institute of Technulugy, Atlanta, Gcorgia.
} 
qualitative effects of the experimental variables on uniformity, deposition rate, and efficiency are generally known by operating CVD engineers. However, the variables are not independent and most hardware is thereforc produced by a trial-and-error adjustment of experimental variables until a satisfactory but not necessarily optimum set of conditions is found for the production of that particular part. Operation is further, plagued by the occasional occurrence of rough nodular growths. The conditions that lead to these nodules are also qualitatively known, but again there seems to be a combination of variables that may produce them with no one vari-. able being controlling. Therefore, one cannot predict definitely if nodules will occur on a part but only if they are likely to occur.

Finally, there is the irritating lack of knowledge as to whether the rate-controlling mechanism in CVD deposits is adsorption, or diffusion, or chemical reaction, or if each controls in different experimental regions.

The tungsten deposition process was selected for examination for two reasons and for two purposes. The reasons were (I) the tungsten process is a relatively simple CVD process with two initial components, and therefore the variables ran be held to a minimum; and (2) lungsien deposition is a very widely used and practical process, so that the result.ts of the study could be of maximum direct benefit to CVD operations. The purposes were to (1) optimize the tungsten deposition process in a chosen system with respect to each of the three response variables: total deposition, deposition efficiency, and thickness uniformity; and (2) attempt to understand the process, including the rate-controlling mechanisms and, if possible, develop a theoretical approach that accurately predicts the engineering response variable from first principlea.

\section{FXXPERIMENTAL APPARATUS}

A schematic of the experimental equipment used in this investigation is shown in Fig. 1. The apparatus was deliberately limited to the simplest equipment possible for the deposition of tungsten. The usual techniques for enhancing thickness uniformity, such as induced temperature gradients, gas preheaters, and mandrel manipulators, were not included. These would 

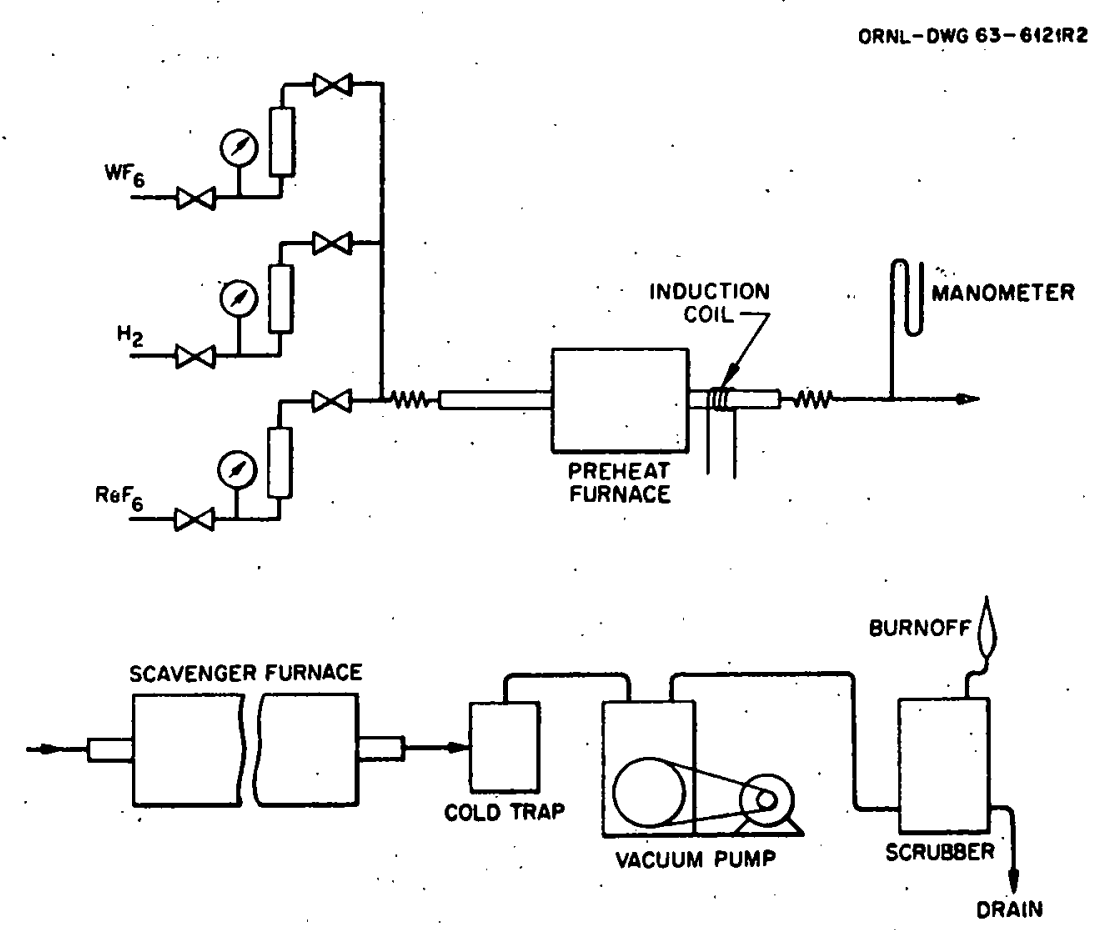

Fig. 1. Deposition Apparatus.

prohibit accurate evaluation of the effects of experimental variables on the deposit homogeneity. The equipment consists of a 16-in. deposition chamber, a scavenger furnace and chamber, a vacuum pump, a cold trap, and a water scrubber to remove hydrogen fluoride. The $\mathrm{WF}_{6}$ flow is monitored by a Hastings flowmeter, but the actual flow rate was calculated by weight change of the $\mathrm{WF}_{6}$ cylinder. The hydrogen flow was measured by a rotameter. The tungsten was deposited in a 0.807-in. -ID copper tube.

\section{STATISTICAL PROGRAM}

The controllable variables of a standard deposition process are temperature, pressure, time, total flow rate, and inlet composition. Time was held constant for all runs in the investigation, and an experimental design was developed to evaluate the effects of the the other variables on chosen response parameters. The design is concerned not with what the response is but only if it is a function of the experimental variables. In the tungsten deposition system the mole fraction of only one component 
is specified to fix the composition of the inlet gas stream. Therefore only four independent variables are needed. The ranges of these four variables are listed below.

$\begin{array}{ll}\text { Temperature, }{ }^{\circ} \mathrm{C} & 450 \text { to } 820 \\ \text { Pressure, torrs } & 9 \text { to } 20 \\ \mathrm{WF}_{6} \text { Flow Rate, } \mathrm{cm}^{3} / \mathrm{min} & 60 \text { to } 370 \\ \mathrm{H}_{2} \text { Flow Rate, } \mathrm{cm}^{3} / \mathrm{min} & 750 \text { to } 5200\end{array}$

The complete design required 81 experiment units, which made up a third-order rotatable design. This allows one to describe the response surface with a cubic polynomial equation. The feature of rotatability guarantees that the precision of the estimates derived frnm the experiment will be unaffected by the orientation of the response surface in the space of the four design variables.

The conditions for the 81 experiments are listed in Tables 1, 2, and 3. The experiments were divided into 3 blocks or phases of 27 exper1-1 ments each. Each block of 27 experiments comprises a complete secondorder rotatable design. Phase 1 covers the extremes in the experimental region, phase 2 the center, and phase 3 the intermediate parts.

\section{PROCEDURE AND EXXERIMENTAL RESULTS}

The experiments were performed in the order specified by the statistical design. The experimental results are given in Tables 4, 5, and 6. We could not achieve some combinations of flow rate and pressure proposed in Table 1 with the available pumping raparity. Also, our Wr6 flow late was determined by a weight differense after the cxpcriment sille lue Hastings flowmeter was not accurate, and we sometimes deviated from the desired tungsten gas ratio.

The deposit thickness of each tube was determined by an x-ray attenuation technique and checked by manual measurement and $x$-ray metallographic techniques. The initial measurements were made at nine points in the 15-in. length, at locations specified by a Gaussian integration technique. Later the $\mathrm{x}$-ray attenuation method was modified to produce a continuous trace of the deposit thickness. Examples of the nine point measurements and of a 1/2-in. increment measurement from a continuous trace are given in Table '?. 
Table 1. Experimental Design frr Investigating Deposition Rates, Phase 1

\begin{tabular}{|c|c|c|c|c|}
\hline Run & $\begin{array}{l}\text { Temperature } \\
\quad\left({ }^{\circ} \mathrm{C}\right)\end{array}$ & $\begin{array}{r}\text { Pressure } \\
\text { (torrs) }\end{array}$ & $\begin{array}{l}\text { Gas Ratio } \\
\left(\% \mathrm{WF}_{6}\right)\end{array}$ & $\begin{array}{l}\text { Flow Rate } \\
\left(\mathrm{cm}^{3} / \mathrm{min}\right)\end{array}$ \\
\hline$i$ & 713.92 & 9.301 & 5.573 & 2405.6 \\
\hline 2 & 635.00 & 12.500 & 6.000 & 3600.0 \\
\hline 3 & 635.00 & 12.500 & 7.000 & 3600.0 \\
\hline 4 & 71.3 .92 & 15.700 & 6.427 & 2405.6 \\
\hline 5 & 713.92 & 9.301 & 6.427 & 2405.6 \\
\hline 6 & 713.92 & 15.700 & 5.573 & 4794.4 \\
\hline 7 & 635.00 & 12.500 & 5.000 & 3600.0 \\
\hline 8 & 713.92 & 15.700 & 6.427 & 4794.4 \\
\hline 9 & $7: 13.92$ & 9.301 & 6.427 & 4794.4 \\
\hline 10 & 635.00 & 12.500 & 6.000 & 3600.0 \\
\hline 11 & 713.92 & 15.700 & 5.573 & 2405.6 \\
\hline 12 & 556.08 & 15.700 & 5.573 & 2405.6 \\
\hline 13 & 556.08 & 9.301 & 6.427 & 4794.4 \\
\hline 14 & 556.08 & 15.700 & 6.427 & 4794.4 \\
\hline 15 & 556.08 & 9.301 & 5.573 & 2405.6 \\
\hline 16 & 635.00 & 12.500 & 6.000 & 6400.0 \\
\hline 17 & 635.00 & 12.500 & 6.000 & 800.0 \\
\hline 18 & 556.08 & 15.700 & 6.427 & 2405.6 \\
\hline 19 & 820.00 & 12.500 & 6.000 & 3600.0 \\
\hline 20 & 556.08 & 9.301 & 6.427 & 2405.6 \\
\hline 21 & 556.08 & 9.301 & 5.573 & . 4794.4 \\
\hline 22 & 635.00 & 20.000 & 6.000 & 3600.0 \\
\hline 23 & 713.92 & 9.301 & 5.573 & 4794.4 \\
\hline 24 & 635.00 & 5.000 & 6.000 & 3600.0 \\
\hline 25 & 556.08 & 15.700 & 5.573 & 4794.4 \\
\hline 26 & 635.00 & 12.500 & 6.000 & 3600.0 \\
\hline 27 & 450.00 & 12.500 & 6.000 & 3600.0 \\
\hline
\end{tabular}


Table 2.: Experimental Design for Investigating Deposition Rates, Phase 2

\begin{tabular}{|c|c|c|c|c|}
\hline Run & $\begin{array}{l}\text { Temperature } \\
\left({ }^{\circ} \mathrm{C}\right)\end{array}$ & $\begin{array}{r}\text { Pressure } \\
\text { (torrs) }\end{array}$ & $\begin{array}{l}\text { Gas Ratio } \\
\left(\% \mathrm{WF}_{6}\right)\end{array}$ & $\begin{array}{l}\text { Flow Rate } \\
\left(\mathrm{cm}^{3} / \mathrm{min}\right)\end{array}$ \\
\hline 1 & 635.00 & $i 2.500$ & 6.000 & 3600.0 \\
\hline 2 & 713.92 & 12.500 & 5.573 & 3600.0 \\
\hline 3 & 713.92 & 12.500 & 6.000 & 4794.4 \\
\hline 4 & 556.08 & 12.500 & 6.000 & 2405.6 \\
\hline 5 & 713.92 & 15.700 & 6.000 & 3600.0 \\
\hline 6 & 635.00 & .75 .700 & 6.427 & 3600.0 \\
\hline 7 & 635.00 & 9.301 & 6.427 & 3600.0 \\
\hline 8 & 635.00 & 12.500 & 6.427 & 2405.6 \\
\hline 9 & 556.08 & 12.500 & 6.000 & 4794.4 \\
\hline 10 & 635.00 & 12.500 & 6.427 & 4794.4 \\
\hline 11 & 635.00 & 12.500 & 5.573 & 2405.6 \\
\hline 12 & 635.00 & 9.301. & 6.000 & 2405.6 \\
\hline 13 & 635.00 & 15.700 & 6.000 & 4794.4 \\
\hline 14. & 635.00 & 12.500 & 6.000 & 3600.0 \\
\hline 15 & 713.92 & 9.301 & 6.000 & 3600.0 \\
\hline 16 & 713.92 & 12.500 & 6.427 & $3600: 0$ \\
\hline .17 & 635.60 & 15.700 & 5.573 & 3600.0 \\
\hline 18 & 556.08 & 15.700 & 6.000 & 3600.0 \\
\hline 19 & 556.08 & 12.500 & 6.427 & 3600.0 \\
\hline 20 & 635.00 & 12.500 & 5.573 & 4794.4 \\
\hline 21 & 635.00 & 9.301 & 6.000 & 4794.4 \\
\hline 22 & 556.08 & $9.3 n T$ & 6.000 & 3600.0 \\
\hline 23 & 635.00 & 9.301 & 5.573 & 3600.0 \\
\hline 24 & 713.92 & 12.500 & 6.000 & 2405.6 \\
\hline 25 & 556.08 & 12.500 & 5.573 & 3600.0 \\
\hline 26 & 635.00 & 15.700 & 6.000 & 2405.6 \\
\hline 27 & 635.00 & 12.500 & 6.000 & 3600.0 \\
\hline
\end{tabular}


Table 3. Experịmental Design for Investigating Deposition Rates, Phase 3

\begin{tabular}{|c|c|c|c|c|}
\hline Run & $\begin{array}{l}\text { Temperature } \\
\left({ }^{\circ} \mathrm{C}\right)\end{array}$ & $\begin{array}{r}\text { Pressure } \\
\text { (torrs) }\end{array}$ & $\begin{array}{c}\text { Gas Ratio } \\
\left(\% \mathrm{WF}_{6}\right)\end{array}$ & $\begin{array}{l}\text { Flow Rate } \\
\left(\mathrm{cm}^{3} / \mathrm{min}\right)\end{array}$ \\
\hline 1 & 507.06 & 12.500 & 6.000 & 1663.7 \\
\hline 2 & 635.00 & 12.500 & 6.692 & 1663.7 \\
\hline 3 & 762.94 & 12.500 & 6.000 & 5536.3 \\
\hline 4 & 635.00 & 7.313 & 5.308 & 3600.0 \\
\hline 5 & 635.00 & 17.687 & 6.692 & 3600.0 \\
\hline 6 & 635.00 & 12.500 & 6.000 & 3600.0 \\
\hline 7 & 635.00 & 12.500 & 6.000 & 3600.0 \\
\hline 8 & 635.00 & 12.500 & 5.308 & 5536.3 \\
\hline 9 & 507.06 & 12.500 & 6.000 & 5536.3 \\
\hline 10 & 762.94 & 12.500 & 5.308 & 3600.0 \\
\hline 11 & 635.00 & 7.313 & 6.692 & 3600.0 \\
\hline 12 & 635.00 & 7.313 & 6.000 & 1663.7 \\
\hline 13 & 635.00 & 17.687 & 5.308 & 3600.0 \\
\hline 14 & 762.94 & 12.500 & 6.692 & 3600.0 \\
\hline 15 & 635.00 & 12.500 & 6.692 & 5536.3 \\
\hline 16 & 507.06 & 7.313 & 6.000 & 3600.0 \\
\hline 17 & 635.00 & 17.687 & 6.000 & 5536.3 \\
\hline 18 & 762.94 & 12.500 & 6.000 & 1663.7 \\
\hline 19 & 507.06 & 12.500 & 5.308 & 3600.0 \\
\hline 20 & 507.06 & $1.7 .68^{\prime} 7$ & 6.000 & 3600.0 \\
\hline 21 & 635.00 & 17.687 & 6.000 & 1663.7 \\
\hline 22 & 762.94 & 7.313 & 6.000 & 3600.0 \\
\hline 23 & 635.00 & 7.313 & 6.000 & 5536.3 \\
\hline 24 & 635.00 & 12.500 & 5.308 & 1663.7 \\
\hline 25 & 762.94 & 17.687 & 6.000 & 3600.0 \\
\hline 26 & 635.00 & 12.500 & 6.000 & 3600.0 \\
\hline 27 & 507.06 & 12.500 & 6.692 & 3600.0 \\
\hline
\end{tabular}


Table 4. Experimental Variables and Results, Phase $1^{a}$

\begin{tabular}{|c|c|c|c|c|c|c|c|c|c|c|}
\hline \multirow{2}{*}{ Run } & \multirow{2}{*}{$\begin{array}{c}\text { System } \\
\text { Pressure } \\
\text { (torrs) }\end{array}$} & \multicolumn{2}{|c|}{$\begin{array}{l}\text { Metered Flow } \\
\left(\mathrm{cm}^{3} / \mathrm{min}\right)\end{array}$} & \multirow{2}{*}{$\begin{array}{r}\mathrm{WF}_{6} \\
\text { Used } \\
(\mathrm{g}) .\end{array}$} & \multirow{2}{*}{$\begin{array}{c}\mathrm{WF}_{6} \\
\mathrm{Flow}^{\mathrm{l}} \\
\left(\mathrm{cm}^{3} / \mathrm{min}\right)\end{array}$} & \multirow{2}{*}{$\begin{array}{c}\text { Available } \\
\text { Tungsten } \\
\text { (g) }\end{array}$} & \multirow{2}{*}{$\begin{array}{c}\text { Total } \\
\text { Depositea } \\
\text { (g) }\end{array}$} & \multirow{2}{*}{$\begin{array}{c}\text { Total } \\
\text { Efficiency } \\
(\%)\end{array}$} & \multicolumn{2}{|c|}{$\begin{array}{r}\text { Deposition in } \\
\text { 15-in. Section }\end{array}$} \\
\hline & & $\mathrm{NF}_{6}$ & $\mathrm{H}_{\tilde{\epsilon}}$ & & & & & & $\begin{array}{l}\text { Weight } \\
\text { (g) }\end{array}$ & $\begin{array}{c}\text { Efficiency } \\
(\%)\end{array}$ \\
\hline 1 & 9.3 & 134 & 2272 & 515 & 129 & 317.9 & 311 & 97.8 & 292 & 91.8 \\
\hline 2 & 12.5 & 216 & 338 & 960 & 241 & 592.6 & 399 & 67.3 & 370 & 62.4 \\
\hline 3 & 12.5 & 252 & 3343 & 1000 & 250 & 617.3 & 397 & 64.3 & 358 & 58.0 \\
\hline 4 & 15.7 & 155 & 2251 & 610 & 153 & 376.5 & 382 & 100.0 & 350 & 92.8 \\
\hline 5 & 9.3 & 155 & 2251 & 640 & 160 & 395.0 & 370 & 93.7 & 349 & 88.3 \\
\hline 6 & 15.7 & 267 & 4527 & 1030 & 258 & 635.8 & $595^{\circ}$ & 93.6 & 561 & .88 .2 \\
\hline 7 & 12.5 & 180 & 3420 & 740 & 155.5 & 456.8 & 394 & 86.2 & 368 & 80.5 \\
\hline 8 & 15.7 & 308 & 4486 & 1200 & 301 & 740.7 & 692 & 93.5 & 673 & 90.8 \\
\hline 9 & 14.0 & 308 & 4486 & 1200 & 301 & 740.7 & 670 & 90.4 & 630 & 85.0 \\
\hline 10 & 12.5 & 216 & 3384 & 950 & 238 & 950.0 & 586 & 69.9 & 378 & 64.0 \\
\hline 11 & 15.7 & 134 & 2272 & 510 & 128 & 314.8 & 308 & 97.8 & 272 & 86.3 \\
\hline 12 & 15.7 & 134 & $227 \bar{z}$ & 525 & 132 & 324.1 & 168 & 51.8 & 153 & 47.2 \\
\hline 13 & 11.3 & 308 & 4486 & 1200 & 301 & 740.7 & 162 & 21.9 & 141 & 19.0 \\
\hline 14 & 15.7 & 308 & 4486 & 1235 & 309 & 762.3 & 170 & 22.3 & 147 & 19.3 \\
\hline 15 & 9.3 & 134 & 2272 & 560 & $1 \angle 0$ & 345.7 & 136 & 39.3 & 122 & 35.3 \\
\hline 16 & 12.5 & 384 & 6016 & 1420 & 356 & 876.5 & 532 & 60.1 & 483 & 55.1 \\
\hline 17 & 12.5 & 48 & 752 & 240 & $\epsilon 0$ & 148.1 & 135 & 91.1 & 127 & 85.8 \\
\hline 18 & 15.7 & 155 & 2251 & 625 & 156 & 385.6 & 193 & 50.0 & 172 & 44.6 \\
\hline 19 & 12.5 & 216 & 3384 & 900 & 225 & 555.5 & 524 & 94.3 & 475 & 85.4 \\
\hline 20 & 9.3 & 155 & 2251 & 600 & 150 & 370.4 & 137 & 37.0 & 115 & 31.1 \\
\hline 21 & 12.3 & 267 & 4527 & 1070 & 265 & 660.5 & 171 & 25.9 & 148 & 22.4 \\
\hline 22 & 20.0 & 216 & 3384 & 925 & 231 & 571.0 & 472 & 82.7 & 441 & 77.2 \\
\hline 23 & $9: 3$ & 267 & 4527 & 1100 & 275 & 680.0 & 609 & 89.6 & 570 & 83.9 \\
\hline 24 & 12.3 & 216 & 3384 & 900 & 225 & 555.5 & 400 & 72.0 & 362 & 65.1 \\
\hline 25 & 15.7 & 267 & 4527 & 995 & 267 & 614.2 & 179 & 29.1 & 158 & 25.7 \\
\hline 26 & 15.3 & 216 & 3384 & 900 & 225 & 555.5 & 440 & 79.2 & 399 & 71.8 \\
\hline 27 & 12.5 & 216 & 3384 & 900 & 225 & 555.5 & 40 & 7.2 & 23 & 4.1 \\
\hline
\end{tabular}


Table 5. Experimental Variables and Results, Phase $2^{a}$

\begin{tabular}{|c|c|c|c|c|c|c|c|c|c|c|}
\hline \multirow{2}{*}{ Run } & \multirow{2}{*}{$\begin{array}{l}\text { System } \\
\text { Pressure } \\
\text { (torrs) }\end{array}$} & \multicolumn{2}{|c|}{$\begin{array}{l}\text { Metered Flow } \\
\left(\mathrm{cm}^{3} / \mathrm{min}\right)\end{array}$} & \multirow{2}{*}{$\begin{array}{l}\text { WF }_{6} \\
\text { Used } \\
\text { (g) }\end{array}$} & \multirow{2}{*}{$\begin{array}{c}\mathrm{WF}_{6} \\
\mathrm{FlOW}^{2} \\
\left(\mathrm{~cm}^{3} / \mathrm{min}\right)\end{array}$} & \multirow{2}{*}{$\begin{array}{l}\text { Available } \\
\text { Tungsten } \\
\text { (g) }\end{array}$} & \multirow{2}{*}{$\begin{array}{l}\text { Total } \\
\text { Ireposited } \\
\text { (g) }\end{array}$} & \multirow{2}{*}{$\begin{array}{c}\text { Total } \\
\text { Eficiciency } \\
(\%)\end{array}$} & \multicolumn{2}{|c|}{$\begin{array}{l}\text { Deposition in } \\
\text { 15-in. Section }\end{array}$} \\
\hline & & $\mathrm{WF}_{6}$ & $\mathrm{H}_{2}$ & & & & & & $\begin{array}{l}\text { Weight } \\
(\mathrm{g})\end{array}$ & $\begin{array}{c}\text { Efficiency } \\
(\%)\end{array}$ \\
\hline 1 & 12.5 & 216 & 3384 & 900 & 225 & 555.5 & 406 & 73.1 & 371 & 66.8 \\
\hline 2 & 12.5 & 200 & 3400 & 840 & 210 & 518.5 & 486 & 93.7 & 461 & 88.9 \\
\hline 3 & 12.5 & 288 & 4506 & 1060 & 266 & 654.3 & 594 & 90.8 & 557 & 85.1 \\
\hline 4 & 12.5 & 150 & 2356 & 540 & 135 & 333.3 & 166 & 49.8 & 147 & 44.1 \\
\hline 5 & 15.7 & 216 & 3384 & 850 & 213 & 524.7 & 516 & 98.3 & 489 & 93.2 \\
\hline 6 & 15.7 & 231 & 3369 & 875 & 219 & 540.1 & 416 & 77.0 & 382 & 70.7 \\
\hline 7 & 9.3 & 231 & 3369 & 900 & 225 & 555.5 & 323 & 58.1 & 296 & 53.3 \\
\hline 8 & 12.5 & 155 & 2251 & 525 & 132 & 324.1 & 308 & 95.0 & 276 & 85.2 \\
\hline 9 & $16.5 \rightarrow 35.0$ & 288 & 4506 & 1100 & 276 & 680.0 & 207 & 30.4 & 183 & 26.9 \\
\hline 10 & 12.5 & 308 & 4486 & 1200 & 301 & 740.7 & 404 & 54.5 & 367 & 49.5 \\
\hline 11 & 12.5 & 134 & 2272 & 525 & 132 & 324.1 & 308 & 95.0 & 292 & 90.1 \\
\hline 12 & 9.3 & 150 & 2356 & 560 & 140 & 345.7 & 315 & 91.1 & 291 & 84.2 \\
\hline 13 & 15.7 & 288 & 4506 & 1215 & 304 & 750.0 & 400 & .53 .3 & 364 & 48.5 \\
\hline 14 & 12.5 & 216 & 3384 & 910 & 228 & 561.7 & 356 & 63.4 & 326 & 58.0 \\
\hline 15 & 13.2 & 216 & 3384 & 880 & 220 & 543.2 & 519 & 95.5 & 484 & 89.1 \\
\hline 16 & 12.5 & 231 & 3369 & 940 & 235 & 580.2 & 544 & 93.8 & 515 & 88.8 \\
\hline 17 & 15.7 & 200 & 3400 & 825 & 206 & 509.2 & 405 & 79.5 & 379. & 74.4 \\
\hline 18 & 15.7 & 216 & 3384 & 875 & 219 & 540.1 & 173 & 32.0 & 159 & 29.4 \\
\hline 19 & 12.5 & 231 & 3369 & 910 & 228 & 561.7 & 161 & 28.9 & 148 & 26.3 \\
\hline 20 & $14.4 \rightarrow 18: 0$ & 267. & 4527 & 1055 & 264 & 651.2 & 501 & 76.9 & 461 & 70.8 \\
\hline 21 & 11.2 & 288 & 4506 & 1120 & 281 & 691.3 & 468 & 67.7 & 433 & 62.6 \\
\hline 22 & 9.6 & 216 & 3384 & 890 & 223 & 549.4 & 137 & 24.9 & 125 & 22.7 \\
\hline 23 & 9.3 & 200 & 3400 & 850 & 213 & 524.7 & 374 & 71.3 & 344 & 65.6 \\
\hline 24 & 12.5 & 150 & 2356 & 550 & 138 & 339.5 & 337 & 99.3 & 312 & 91.9 \\
\hline 25 & 12.5 & 200 & 3400 & 875 & 219 & 540.1 & 173 & 32.0 & 156 & 28.9 \\
\hline 26 & 15.7 & 150 & 2356 & 525 & 132 & 324.1 & 323 & 99.7 & 306 & 94.4 \\
\hline 27 & 12.5 & 216 & 3384 & 930 & 233 & 574.1 & 379 & 66.0 & 346 & 60.3 \\
\hline
\end{tabular}

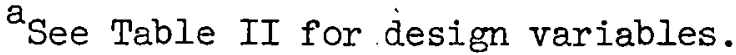


Table 6. Experimental Variables and Results, Phase $3^{a}$

\begin{tabular}{|c|c|c|c|c|c|c|c|c|c|c|}
\hline \multirow{2}{*}{ Run } & \multirow{2}{*}{$\begin{array}{l}\text { System } \\
\text { Pressure } \\
\text { (torrs) }\end{array}$} & \multicolumn{2}{|c|}{$\begin{array}{c}\text { Metered Flow } \\
\left(\mathrm{cm}^{3} / \mathrm{min}\right)\end{array}$} & \multirow{2}{*}{$\begin{array}{r}\mathrm{WF}_{6} \\
\text { Used } \\
(\mathrm{g})\end{array}$} & \multirow{2}{*}{$\begin{array}{c}\mathrm{WF}_{6} \\
\mathrm{FlOW}^{\mathrm{F} \mathrm{O}^{-}} \\
\left(\mathrm{sm}^{3} / \mathrm{min} !\right.\end{array}$} & \multirow{2}{*}{$\begin{array}{c}\text { Available } \\
\text { Tungsten } \\
\text { (g) }\end{array}$} & \multirow{2}{*}{$\begin{array}{c}\text { Total } \\
\text { Deposited } \\
\text { (g) }\end{array}$} & \multirow{2}{*}{$\begin{array}{c}\text { Total } \\
\text { Efficiency } \\
(\%)\end{array}$} & \multicolumn{2}{|c|}{$\begin{array}{l}\text { Deposition in } \\
\text { 15-in. Section }\end{array}$} \\
\hline & & $\mathrm{WF}_{6}$ & $\mathrm{H}_{2}$ & & & & & & $\begin{array}{l}\text { Weight } \\
\text { (g) }\end{array}$ & $\begin{array}{l}\text { Efficiency } \\
(\%)\end{array}$ \\
\hline 1 & 12.5 & 100 & 1564 & 445 & 111 & 274.7 & 88 & 32.0 & 76 & 27.7 \\
\hline 2 & 12.5 & 111 & 1553 & 475 & 119 & 293.2 & 279 & 95.1 & 261 & 89.0 \\
\hline 3 & 14.0 & 332 & 5204 & 1325 & 332 & 817.9 & 734 & 89.7 & 689 & 84.2 \\
\hline 4 & 10.6 & 191 & 3409 & 700 & 225 & 555.5 & 363 & 65.3 & 331 & 59.6 \\
\hline 5 & 17.6 & 241 & 3359 & 1200 & 300 & 740.2 & 500 & 67.5 & 458 & 61.9 \\
\hline 6 & 12.5 & 216 & 3384 & 900 & 225 & 555.5 & 405 & 72.9 & 374 & 67.3 \\
\hline 7 & 12.5 & 216 & 3384 & 825 & 207 & 509.2 & 391 & 76.8 & 349 & 68.5 \\
\hline 8 & 13.4 & 294 & 5242 & 1075 & 269 & 663.6 & & & 397 & 59.8 \\
\hline 9 & 14.0 & 332 & 5204 & 1225 & 307 & 756.1 & 175 & 23.1 & 149 & 19.7 \\
\hline 10 & 12.5 & 191 & 3409 & 775 & 194 & 478.4 & 474 & 99.1 & 440 & 92.0 \\
\hline 11 & 9.4 & 241 & 3359 & 975 & 244 & 601.8 & 354 & 58.8 & 324 & 53.8 \\
\hline 12 & 7.3 & 100 & 1564 & 475 & 119 & 293.2 & 254 & 86.6 & 233 & 79.5 \\
\hline 13 & 17.7 & 191 & 3409 & 845 & 211 & 521.6 & 442 & 84.7 & 414 & 79.4 \\
\hline 14 & 12.5 & 241 & 3359 & 925 & 232 & 571.0 & 543 & 95.1 & 509 & 89.1 \\
\hline 15 & 14.5 & 370 & 5166 & 1335 & 335 & 824.0 & 410 & 49.7 & 372 & 45.1 \\
\hline 16 & 9.0 & 216 & 3384 & 865 & 217 & 533.9 & 64 & 12.0 & 56 & 10.5 \\
\hline 17 & 17.7 & 332 & 5204 & $1 \approx 25$ & 307 & 756.1 & 450 & 59.5 & 412 & 54.5 \\
\hline 18 & 12.5 & 100 & 1564 & $\angle 75$ & 119 & 293.2 & 264 & 90.0 & 218 & 74.3 \\
\hline 19 & 12.5 & 191 & 3409 & r75 & 194 & 478.4 & 84 & 17.5 & 75 & 15.7 \\
\hline 20 & 17.7 & 226 & 3384 & $\subseteq 00$ & 225 & 555.5 & 95 & 17.1 & 84 & 15.1 \\
\hline 21 & 17.7 & 100 & 1564 & 450 & 113 & 277.8 & 274 & 98.6 & 256 & 92.1 \\
\hline 22 & 8.8 & 216 & 3384 & 875 & 219 & 540.1 & 515 & 95.3 & 483 & 89.4 \\
\hline 23 & 14.0 & 332 & 5204 & 1275 & 320 & 787.0 & 415 & 52.7 & 386 & 49.0 \\
\hline 24 & 12.5 & 88 & 1576 & 400 & 100 & 246.9 & 235 & 95.2 & 218 & 88.3 \\
\hline 25 & 17.6 & 216 & 3384 & 850 & 213 & 524.7 & 506 & 96.4 & 464 & 88.4 \\
\hline 26 & 12.5 & 216 & 3384 & 850 & 213 & 524.7 & 405 & 77.2 & 374 & 71.3 \\
\hline 27 & 12.5 & 241 & 3359 & 1025 & 256 & 632.7 & 90 & 14.2 & 81 & 12.8 \\
\hline
\end{tabular}

${ }^{a}$ See Table III for design rariables. 
Table 7. Deposit Thickness as a Function of Position Along Mandrelsa

Distance from Section End.
(in.)

Measured Values ${ }^{b}$

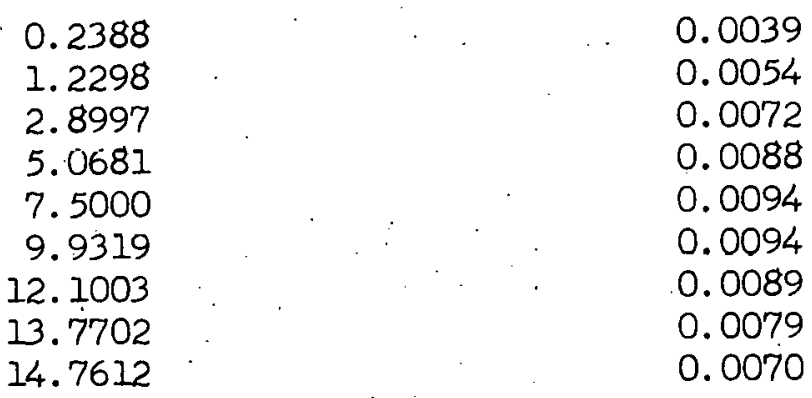

Read from Continuous Scan
0.5000
0.0044
1.0000
0.0051
1. 5000
0.0058
2.0000
0.0064
2.5000
0.0069
3.0000
0.0073
3.5000
0.0078
4.0000
0.0081
4.5000
0.0085
5.0000
0.0088
5.5000
0.0090
6.0000
0.0091
6.5000
0.0093
7.0000
0.0093
7.5000
0.0094
8.0000
0.0094
8.5000
0.0095
9.0000
0.0095
9. 5000
0.0095
10.0000
0.0094
10.5000
0.0094
11.0000
0.0093
11.5000
0.0092
12.0000
0.0090
13.0000
0.0085
13.5000
0.0082
14.0000
0.0078
14.5000
0.0073
15.0000
0.0068

$a_{\text {From phase 3, run } 27 .}$

bor statistically selected locations. 


\section{STATISTICAL RESULTS}

Initially the three response variables were correlated and plotted as functions of the four experimental variables selected according to two models. Along with temperature and pressure, model 1 used total flow rate and $W_{6}$ percentage in the inlet gas, while model 2 used flow rates of the two reactant gases. Each response was measured for the center 15 in. of the 16-in. mandrel. Typical graphs of these responses are plotted in Figs. 2 through 7.

Figure 2 shows an example of the total deposition pressuretemperature contours calculated accuriing to modcl 1 . It is one graph from a sequence in which total flow rate and $\mathrm{WF}_{6}$ percentage were varied. One could similarly plot the response contours as functions of any two experimental parameters for selected values of the other two; temperature

QRML=OWO $67-5691$

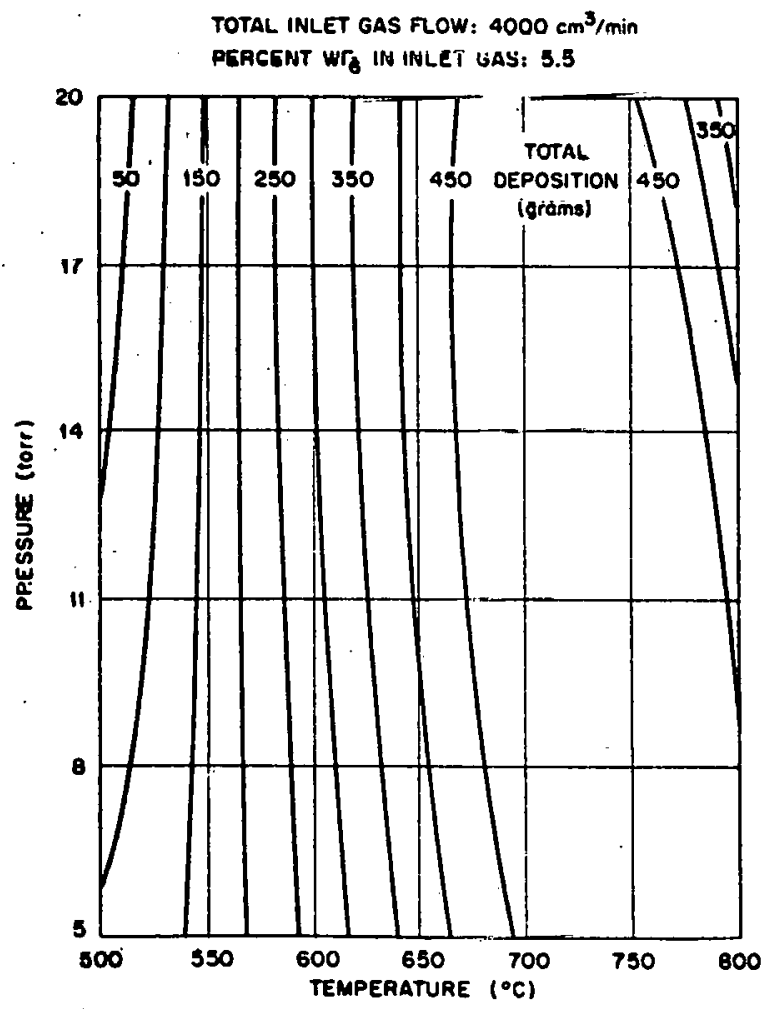

Fig. 2. Total Deposition Contours for a Total Inlet Gas Flow of $4000 \mathrm{~cm}^{3} / \mathrm{min}$ and $5.5 \% \mathrm{WF}_{6}$ in the Inlet Gas. 


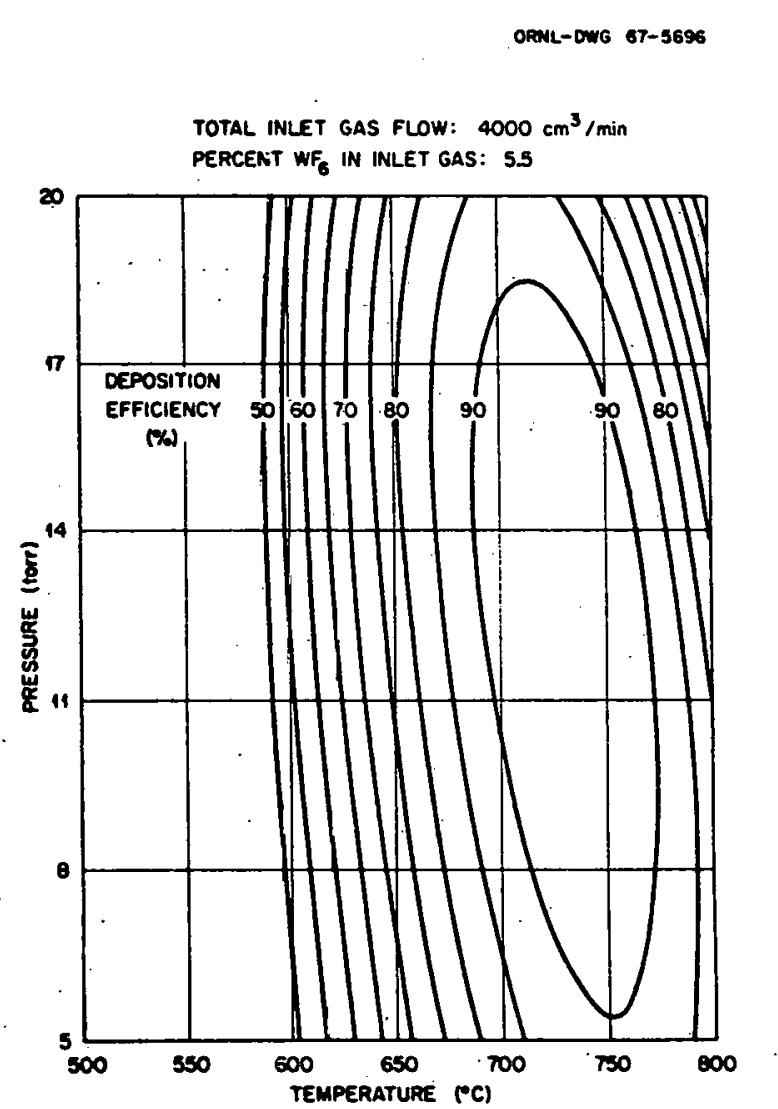

Fig. 3. Total Deposition Contours for Inlet Flow Rates of $2500 \mathrm{~cm}^{3} /$ min $\mathrm{H}_{2}$ ard $200 \mathrm{~cm}^{3} / \mathrm{min}_{6}$.
INLET HYOROGEN FLOW: $2500 \mathrm{~cm}^{3} / \mathrm{min}$ INLET $W F_{G}$ FLOW: $200 \mathrm{~cm}^{3} / \mathrm{min}$

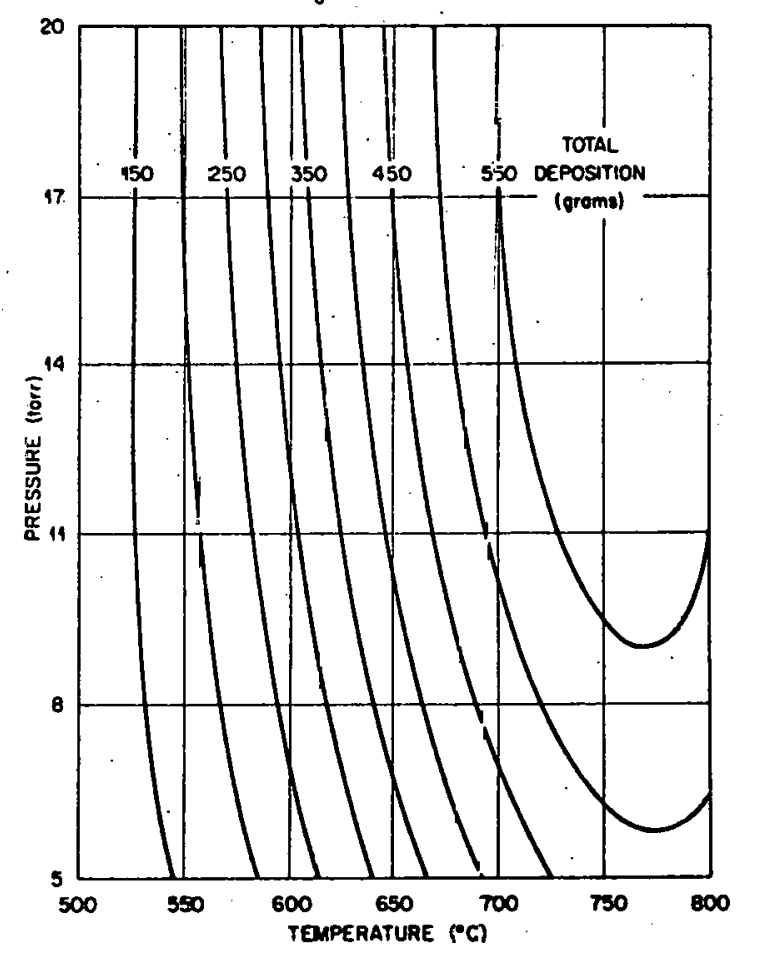

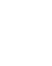


ORML-DW6 67-5.695

NLET HYOROGEN FLOW: $2300 \mathrm{~cm}^{3} / \mathrm{min}$ TNLET WF FLOW: $200 \mathrm{~cm}^{3} / \mathrm{min}$

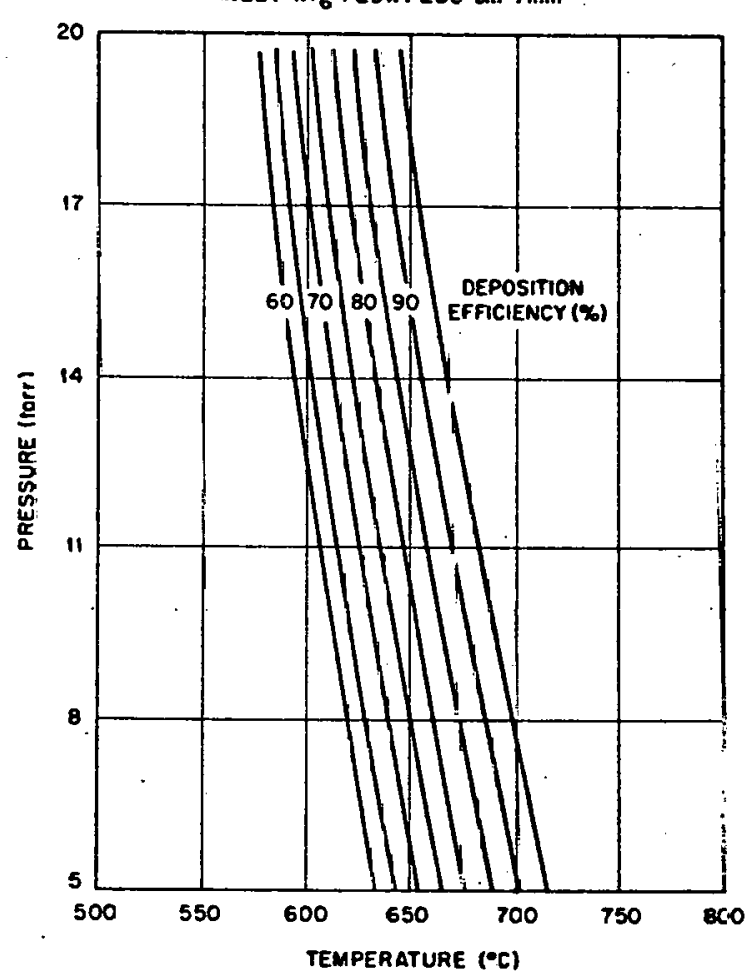

Fig. 5. Deposition Efficiency Contours fer Inlet FlDw Rates of $2500 \mathrm{~cm}^{3} / \mathrm{min} \mathrm{H}_{2}$ and $200 \mathrm{~cm}^{3} / \min W_{6}$.
ORNL-DW6 67-5694

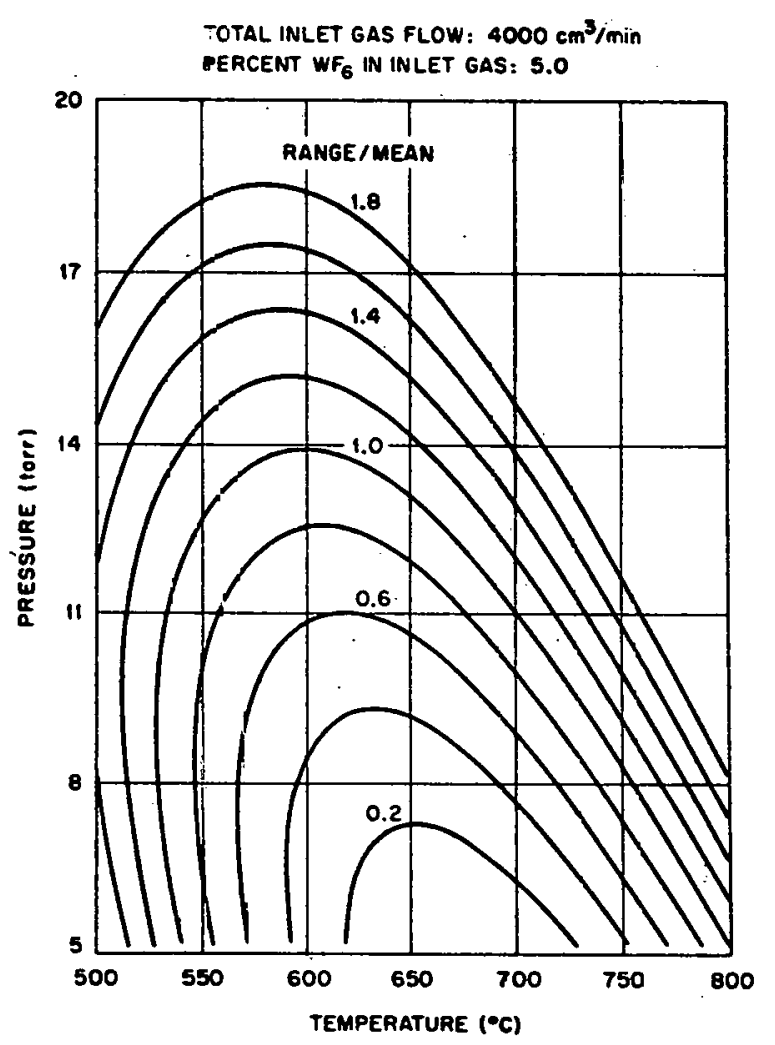

Fig. 6. Range-to-Mean Thickness Ratio Contours for a Total Inlet Gas Flow of $4000 \mathrm{~cm}^{3} / \mathrm{min}$ anc $5.0 \% \mathrm{WF}_{6}$ in the Inlet Gas. 


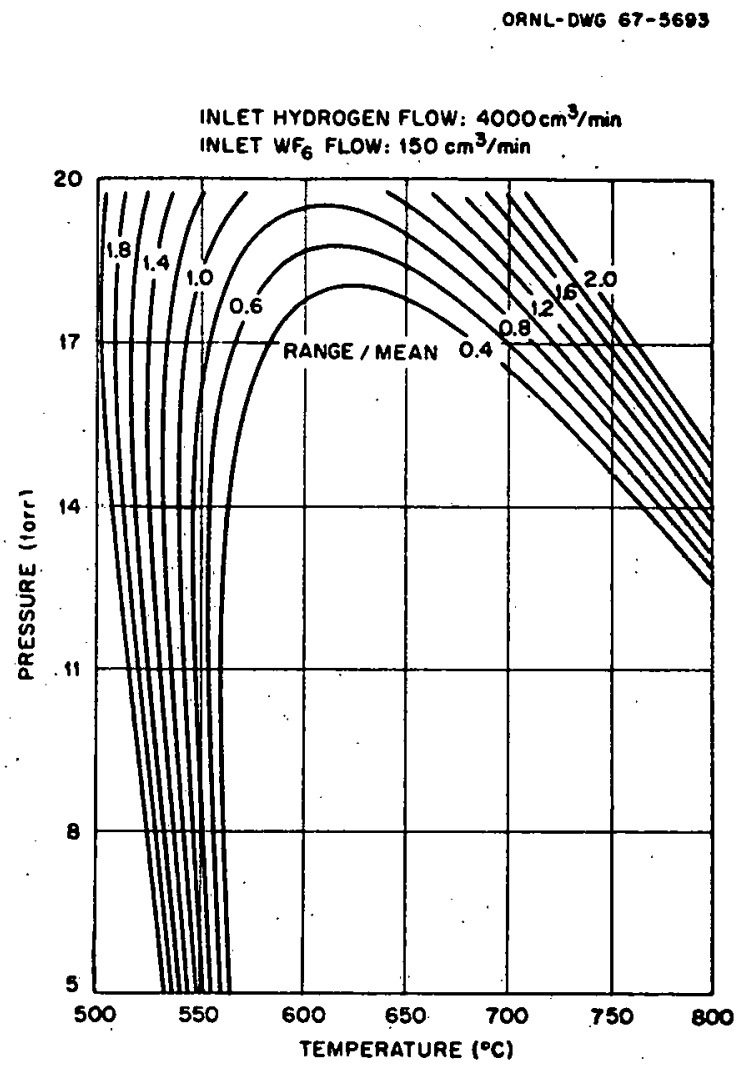

Fig. 7. Range-to-Mean Thickness Ratio Contours for Inlet Gas Flow of $4000 \mathrm{~cm}^{3} / \mathrm{min} \mathrm{H}_{2}$ and $150 \mathrm{~cm}^{3} / \mathrm{min} \mathrm{WF}_{6}$.

and pressure are used in these examples since they are the most completely independent variables in our system. Figure 3 shows a similar example of total deposition plotted according to model 2 . Figures 4 and 5 show examples of the deposition efficiency calculated for the two models. This quantity is the weight of deposit in the 15-in. length expressed as a percentage of the weight of tungsten introduced as $\mathrm{WF}_{6}$. Figures 6 and 7 illustrate the range of deposition thickness in the 15-in. length divided by the mean deposition thickness in that length, plotted for both models.

Up to this point, all our correlations are with the inlet gas conditions. As the gas reacts passing through the mandrel, its composition and flow rate vary; $\mathrm{WF}_{6}$ and hydrogen are consumed and hydrogen fluoride appears. With a simple stoichiometric balance, the average compositiun and flow rate at any point can be calculated from the 
initial conditions and the mass of tungsten deposited up to that point. Applying a Gaussian integration technique to the thickness data, we calculated the mass of tungsten deposited up to each of nine selected points along the mandrel. From.it, in turn we calculated the average composition of the gas stream and the component flow rates at each point. Finally, by interpolation we obtained the average gas composition, flow rate, mandrel position, and deposit thickness at points at which the hydrogen fluoride mole fraction in the gas had reached $0.02,0.05,0.10$, and 0.20. The thicknesses obtained were checked by comparison with the continuous scan data. Since the experiments were all for the same duration, the deposit thickness is a moarurc of the pulnt deposition rate. We correlated these with the experimental values, calculated for the extent of reaction along the mandrel. Figure 8 shows an example of this type of correlation.

ORNL-DWG 67-5692

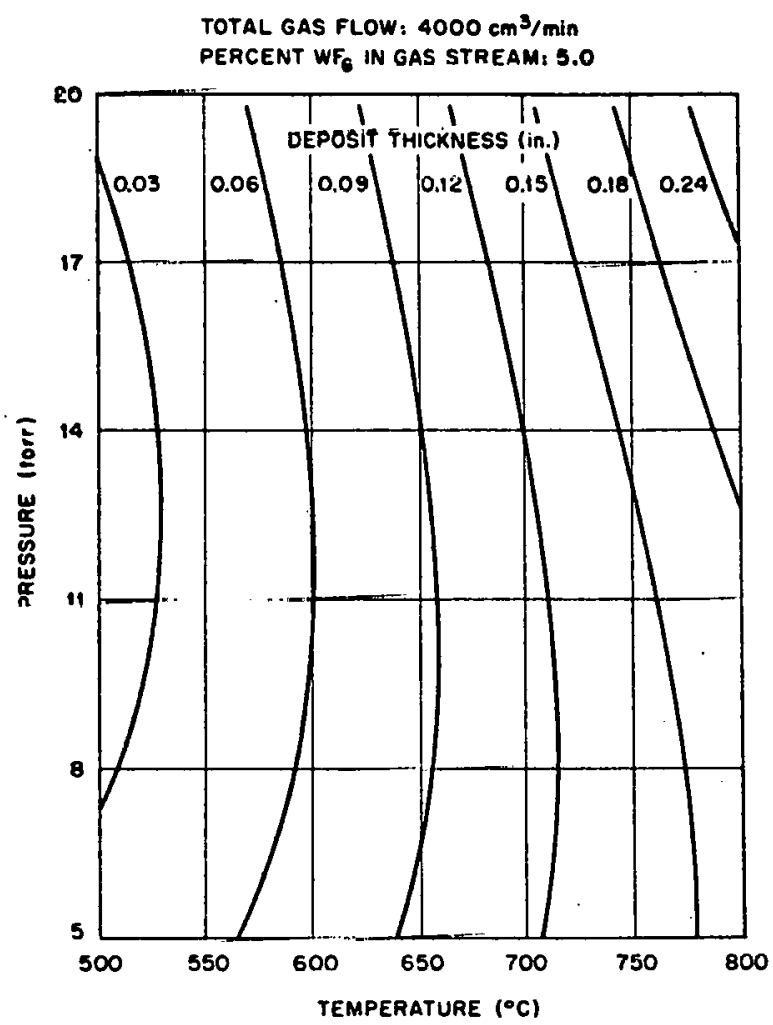

Fig. 8. Deposit Thickness Contours for a Total Gas Flow of $4000 \mathrm{~cm}^{3} / \mathrm{min}$ and $5.0 \% \mathrm{WF}_{6}$ in the Gas Stream at Points Where the Hydrogen Fluoride Mole Fraction Reached 0.05 . 


\section{DISCUSSION AND INTERPRETATION}

The total weight of deposit, deposition efficiency, and thickness uniformity all depend on the length of the heated substrate and are therefore not directly relatable to deposition mechanisms. Their value is that they represent a correlation between controllable experimental variables and the deposit product. Even though the results are not quantitatively applicable to another system of different size or geometry, they show a trend with experimental conditions that should be independent of the system used. Thus, with our results available, a very few experiments with another apparatus should suffice to establish the relationships for that apparatus reasonably well. We believe that systems of a different size but similar geometry will have an analogous correlation between deposit and experimental variables. Therefore, a complete set of the curves generated in this correlation between the listed responses and experimental variables will be published. ${ }^{3}$

Examination of diffusion and laminar flow theory indicates that the curves cannot be applied directly even to systems of cylindrical geometry if they are of a different size. Even though one can introduce exactly the same mass velocity of each component and the same $\mathrm{H}_{2}$-to- $\mathrm{WF}_{6}$ ratio, the curves will not be quantitatively analogous because the response parameters will be a function of the physical dimensions and scallng parameters (e.g., the Reynolds Number and total length-to diameter ratio).

One further observation is necessary in regard to theoretical consideration of these "engineering" curves. Most of the curves with total deposition or efficiency as a response show a double-valued function. The theoretical equations presently derived do not predict or even allow a double-valued function, although our data definitely are double-valuer. This behavior is a function of the apparatus and not a fundamental phenomenon. Any other than an ideal system will have the same characteristics. The double-valued function results from predeposition

${ }^{3}$ F. H. Patterson and W. C. Robinson, Jr., Statistically Designed Chemical-Vapor Deposition Experiment, (to be published). 
in the mandrel ahead of the measured 15-in. length at the higher mandrel temperatures. At temperatures around $700^{\circ} \mathrm{C}$ the observed efficiences in the 15-in. section begin to approach 100\%. At higher mandrel temperatures the gas lines leading into the mandrel become hot enough (despite thermal shielding) to allow deposition, and deposition begins in them. This reduces the apparent efficiency or total deposition in the 15-1n. length, although all the available tungsten is deposited, and creates the double-valued function. Therefore, to optimize efficiency on a given piece of hardware requires a mandrel temperature in the range indicated or the use of special heating or injection techniques.

The deposition thicknesses at hydrogen fluoride concentrations of $0.02,0.05$, and 0.1 mole fraction of the gas stream monotonically increase with hydrogen fluoride concentrations at all values of temperature and pressure.

There were not enough data at 0.20 mole fraction to correlate meaningfully. The monotonic increase is contrary to theory and is believed to be caused by the temperature gradient imposed on the mandrel by the inlet gas stream. The fact that this temperature gradient, which should be small in our apparatus, is still the rate-controling factor at hydrogen fluoride concentrations as high as 0.10 mole fraction indicates that the hydrogen fluoride effect on the deposition rate is small. ubviously the correlation needs to be made at higher hydrogen fluoride concentrations if possible and at low flow rates to minimize the effect of cooling by the inlet gas. Curves showing this are being prepared but are not available at the time of this writing. Since the present data indicate that there is a temperature gradient effect. in the mandrel up to hydrogen fluoride concentrations of 0.1 mole fraction, the usual activation energy plots of deposition rate vs the reciprocal of the absolute temperature were not made from Fig. 8 and/or the other graphs of this series. The temperature was measured on the outside of the copper mandrel near the center of the 15-in. section, so it would not ind1cate accurately the gas-phase temperature until the internal gradient no longer existed. The surprising length of thermal gradient here means that previous Arrhenius-type plots for determination of activation energy may be in error. 
To understand the data generated by this program, we are trying to derive an equation for predicting deposition efficiencies from theoretical considerations. To keep the number of variables at a minimum as well as to obtain a simple closed-form analytical expression, several simplifying assumptions were made. This approach was considered reasonable, since only the form of the equation was desired and not an accurate analytical prediction of deposition efficiency. The vapor deposition process is assumed to involve at least five different steps, ${ }^{4}$ any one of which could govern the rate of deposition. These processes are (1) diffusion of the reactants to the heated surface, (2) adsorption of reactants onto the surface, (3) reaction at the surface, (4) desorption of gaseous products from the surface, and (5) diffusion of gaseous products into the gas stream. The present equation considers only the processes of diffusion and kinetics, with the further assumption that the reaction is first order with respect to $\mathbf{W F}_{6}$. The derivation involves a mass balance, which includes the effect of the decreasing bulk mass fraction of $\mathrm{WF}_{6}$ as well as diffusion to the tube wall and subsequent reaction. The equation is

$$
E=1-\exp -\left\{\frac{C_{1}}{Q_{W F_{6}}+2 Q_{\mathrm{H}_{2}} / 298}\left[1-\frac{1}{1+(P / T w) \exp \left(C_{2}-C_{3} / T_{w}\right)}\right]\right\}
$$

where

$$
\begin{aligned}
\mathrm{E} & =\text { deposition efficiency as a fraction, } \\
\mathrm{Q}_{\mathrm{WF}_{6}} & =\mathrm{WF}_{6} \text { flow rate }\left(\mathrm{cm}^{3} / \mathrm{min}\right) \\
\mathrm{Q}_{\mathrm{H}_{2}} & =\mathrm{H}_{2} \text { flow rate }\left(\mathrm{cm}^{3} / \mathrm{min}\right) \\
\mathrm{p} & =\text { measured system pressure (torrs), } \\
\mathrm{TW}_{\mathrm{w}} & =\text { measured system deposition temperature }\left({ }^{\circ} \mathrm{K}\right) .
\end{aligned}
$$

The details of this derivation will be presented at a later date.

${ }^{4} \mathrm{~J}$. D. Fleming, W. C. Robinson, Jr., and C. F. Leitten, Jr., Thermochemical Analysis of Vapor Plating Processes, ORNL-TM-1454 (July 1966). 
The applicability of this equation was tested by obtaining a leastsquares estimate for the constants $C_{1}, C_{2}$, and $C_{3}$ using all 81 data points. The results of this approach are:

\begin{tabular}{ccc} 
Constant & $\begin{array}{c}\text { Least-Squares } \\
\text { Estimate }\end{array}$ & \multicolumn{2}{c}{$\begin{array}{c}\text { Approximate } \\
\text { Standard Error }\end{array}$} \\
\cline { 2 - 3 } $\mathrm{C}_{1}$ & 788 & 102 \\
$\mathrm{C}_{2}$ & 20.2 & 1.2 \\
$\mathrm{C}_{3}$ & 15,120 & 897
\end{tabular}

An idea of the fit of Eq. (1) may be obtained from Fig. 9, which is a plot of a.1 of the data taken for a pressure of 12.5 torrs and a temperature of $635^{\circ} \mathrm{C}$. The solid curve is a plot of $\mathrm{Eq}$. (1) for a hydrogen flow rate of $3350 \mathrm{~cm}^{3} / \mathrm{min}$. Some of the data points do not correspond to this hydrogen flow rate but were plotted also, since Eq. (I) indicates that for the analytical model chosen the efficiency is not sensitive to the hydrogen flow rate. It is well to keep in mind that the solid curve shown in this figure was obtained using Eq. (1) with the constants $C_{1}$, $\mathrm{C}_{2}$, and $\mathrm{C}_{3}$ obtained by a statistical analysis of all of the data.

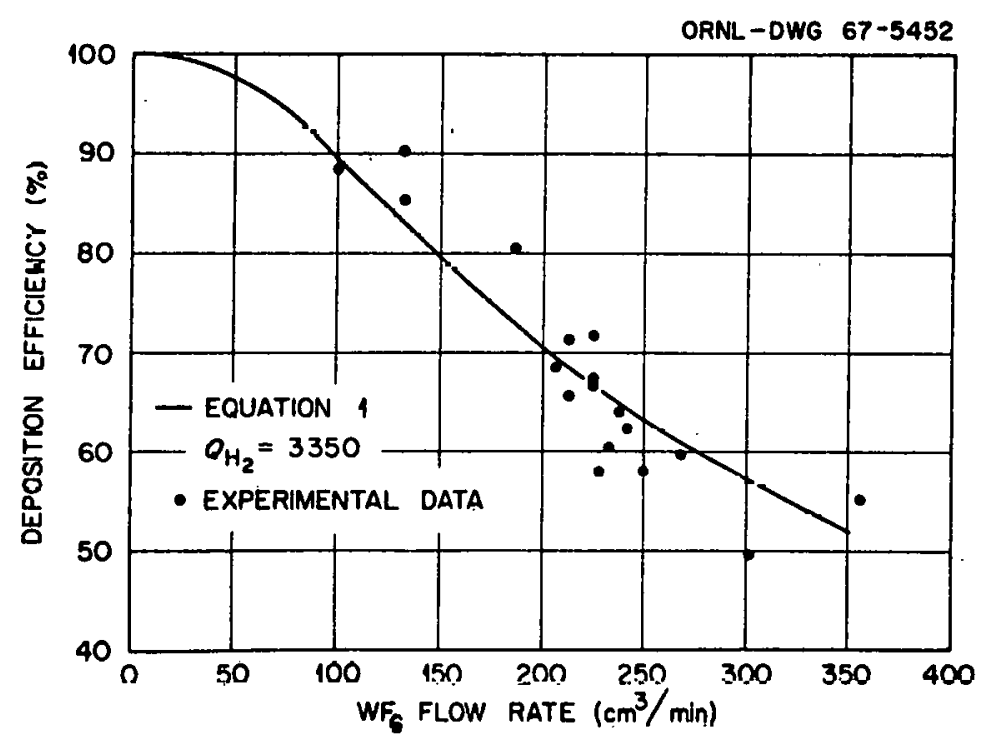

Fig. 9. Deposition Efficiency Vs $\mathrm{WF}_{6}$ Flow Rate. 


\section{CONCLUSIONS AND FUTURE WORK}

The effects of the experimental variables on three response variables, total deposition, deposition efficiency, and deposit thickness uniformity, were defined for deposition of tungsten inside a cylindrical mandrel of 0.807-in. ID. These complete results are presented elsewhere. The same evaluation will be made using other response parameters including mechanical properties, fluorine content, and microstructure. One cannot apply the obtained results directly to other.sizes or shapes, but an attempt will be made to define the relationship between the experimental varlable effects on responses in different sizes and geometries.

The effect of concentration of hydrogen fluoride byproduct on deposition rate is quite small compared to the temperature effect. A long thermal gradient is present in standard deposition mandrels. To evaluate the hydrogen fluoride effect on deposition rate, we will have to examine hydrogen fluoride mole fractions higher than 0.10.

An equation that was developed from theoretical considerations is a reasonable representation of the data in our experimental range. This indicates that diffusion and kinetics are the rate-controlling processes in most of our experimental range. Application of equations developed from considerations of separate rate-controlling processes indicates that diffusion is the controlling process in the low-pressure hightemperature region of our experimental range. The constants in the present equation will be refined by a fit to only the data in the range expected to be' controlled by diffusion or kinetics, and an absorption term will be added to the equation.

\section{ACKNOWLEDGMENITS}

The authors would like to acknowledge the very capable and consistent statiatical design and direstion of D. A. Gardiner and T. L. Hebble, the programming of Sandra Merriman, the experimentation of E. R. Turnbill, and the advice and counsel of W. R. Martin, John Burson, and G. M. Adamson, $\mathrm{J} \mathrm{r}$. 


\section{THIS PAGE}

\section{WAS INTENTIONALLY \\ LEFT BLANK}


ORNL-TM-1890

\section{INTERNAL DISTRIBUTION}

1-3. Central Research Library

4-5. ORNL - Y-12 Technical Library Document Reference Section

6-20. Laboratory Records Department

21. Laboratory Records, ORNL R.C.

22. ORNL Patent Office

23. G. M. Adamson, Jr.

24. G. E. Boyd

25. F. L. Culler

26. J. E. Cunningham

27. C. W. Dean

28. J. H. DeVan

29. R. G. Donnelly

30. J. I. Federer

31. J. H Frye, Jr.
32. W. O. Harms

33-35. M. R. Hill

36. J. Mi Jones

37. C. F. Leitten, Jr.

38. T. S. Lundy

39. H. G. MacPherson

40. W. R. Martin

41. R. E. McDonald

42. D. L. McElroy

43. P. Patriarca

44. G. A. Reimann

45-49. W. C. Robinson

50. A. C. Schaffhauser

51. S. H. Smiley $(\mathrm{K}-25)$

52. R. L. Stephenson

EXTTERNAL DISTRIBUTION

53. J. H. Burson, Georgia Institute of Technology

54. D. F. Cope, RDT, SSR, AEC, Oak Ridge National Laboratory

55. J. D. Fleming, Georgia Institute of Technology

56. C. W. Gorton, Georgia Institute of Technology

57. J. L. Gregg, Bard Hall, Cornell University

58. R. L. Heestand, Battelle Memorial Institute, Columbus, Ohio

59. W. J. Larkin, AEC, Oak Ridge Operations

60. F. H. Patterson, Battelle Memorlal Institute, Columbus, Ohio

61. L. W. Roberts; Lawrence Radiation Laboratory

62. J. M. Simmons, AEC, Washington

63. J. E. Spruiell, the University of Tennessee

64. E. E. Stansbury, the University of Tennessee

65. M. J. Whitman, AEC, Washington

66. Research and Development, AEC, Oak Ridge Operations

67-81. Division of Technical Information Extension 